\title{
Mycorrhizal inoculation modulates root morphology and root phytohormone responses in trifoliate orange under drought stress
}

\author{
Jin Liü,2, Cha Guo ${ }^{1,2}$, Zi-Li Chen ${ }^{1,2}$, Jia-Dong He ${ }^{1,2}$, Ying-Ning Zou1,2* \\ ${ }^{1}$ College of Horticulture and Gardening, Yangtze University, Jingzhou, Hubei 434025, People's Republic of China; ${ }^{2}$ Institute of Root Biology, \\ Yangtze University, Jingzhou, Hubei 434025, People's Republic of China
}

\section{A B S TR A C T}

\begin{abstract}
Phytohormones can be responsible for activating tolerance responses of drought stress (DS). The present study was done to evaluate the effects of an arbuscular mycorrhizal fungus (AMF), Funneliformis mosseae, on root morphology and root phytohormones levels in trifoliate orange (Poncirus trifoliata) seedlings exposed to well-watered (WW, 75\% of maximum water holding capacity) and drought stress ( $55 \%$ of maximum water holding capacity). The six-week DS treatment strongly restricted root mycorrhizal colonization by $27.7 \%$. The DS treatment caused the decrease of total plant biomass and root morphological traits, but the AMF inoculation significantly increased total plant biomass and root total length, projected area, surface area, average diameter, volume, and number of $1^{\text {st }}, 2^{\text {nd }}$, and $3^{\text {rd }}$ lateral root under WW and DS conditions. AMF plants exhibited significantly higher leaf water potential than non-AMF plants exposed to WW and DS. AMF colonization notably regulated the changes in root phytohormone levels: the increase of indole-3-acetic acid (IAA), abscisic acid (ABA), methyl jasmonate (MeJA), and zeatin riboside (ZR) levels under WW condition, and the increase of IAA, ABA, MeJA, ZR, and brassinosteroids (BRs) concentrations under DS conditions. These results concluded that AMF enhanced drought tolerance in trifoliate orange through modulation of root phytohormones and root morphology.
\end{abstract}

Keywords: Abscisic acid; Citrus; Hormone; Indole-3-acetic acid; Mycorrhiza; Aeatin riboside

\section{INTRODUCTION}

Soil drought stress (DS) is one of the important abiotic stresses, strongly limiting crop growth and yield (Nazar et al., 2015). Roots are directly in touch with soil moisture changes, and thus, the damage of plant to drought is firstly in roots. Since plant root is the most crucial organ for water and nutrient absorption, root morphological and physiological responses to DS are an important adaptation to drought (Shan et al., 2015). Hence, evaluating the changes of root physiology and morphology under DS seems to be very important.

Arbuscular mycorrhiza (AM) is a mutualistic symbiosis between arbuscular mycorrizal fungi (AMF) and the roots of $80 \%$ of terrestrial plants. AM develops well extraradical mycelium spread in the soil around the root to absorb nutrient elements and water from the soil to the fungal partner (Johnson and Gibert, 2015; Zhang et al., 2015). The
AMs not only increase plant growth, but also enhance plant tolerance to abiotic stress, such as DS (Wu et al., 2013). In roots of pistachio plants under DS, inoculation with Glomus etunicatum did not alter soluble sugar and proline contents and catalase activity, but significantly increased peroxidase activity and soluble protein concentration (Abbaspour et al., 2012). In two maize cultivars, AMF colonization significantly increased root nitrate reductase activity under the DS (Subramanian and Charest, 1998). These results suggest diverse responses of root physiological activity to AMF under DS conditions.

Plants develop a range of phytohormones to decrease the damage of DS (Diego et al., 2015). Auxin, cytokinins (CKs), gibberellins (GAs) and brassinosteroids (BRs) are defined as "positive growth regulators", and abscisic acid (ABA), jasmonic acid (JA), salicylic acid (SA) and ethylene are counted as "stress hormones" (Kovosá et al., 2012). These phytohormones are thought to be the most important

\footnotetext{
*Corresponding author:

Ying-Ning Zou, College of Horticultura and Gardening, Yangtze University, Jingzhou, Hubei 434025, People's Republic of China.

E-mail: zouyingning@163.com
}

Received: 29 November 2015;

Revised: 16 February 2016;

Accepted: 16 February 2016;

Published Online: 28 February 2016 
signal substances in modulating physiological responses for stress adaptation, such as growth, development, source/ sink transitions and nutrient allocation (Fahad et al., 2014). Therefore, they were important for understanding AM functions on drought tolerance of the host plant. A small amount of studies indicated that AMF colonization significantly increased root zeatin riboside (ZR) levels in flax and ethylene levels in papaya roots under DS, whereas did not alter the root ABA concentration in Medicago sativa (Drüge and Schönbeck, 1992; Giocoechea et al., 1997; Cruz et al., 2000).

Citrus is one of the most important trees in the world including China (Wang et al., 2014). Citrus trees in China often suffer from soil drought stress, resulting in the decline of citrus quality and yield. The aim of this study was to investigate the AMF effects on root morphology and root phytohormone levels of trifoliate orange (Poncirus trifoliata L. Raf.) under DS and WW conditions.

\section{MATERIALS AND METHODS}

\section{Experimental set-up and design}

Seeds of trifoliate orange were surface sterilized by $75 \%$ alcohol solution and germinated in a plastic box containing sterilized $\left(0.11 \mathrm{Mpa}, 121^{\circ} \mathrm{C}, 2 \mathrm{~h}\right)$ sand. After $\sim 3$ weeks, a five-leaf-old seedling was planted into a $19.5 \times 14 \times 21 \mathrm{~cm}$ (upper diameter $\times$ bottom diameter $\times$ height) plastic pot. Soil: Sand $(2: 1, \mathrm{v} / \mathrm{v})$ mixture was used as a growth substrate after autoclaved (0.11 Mpa, $\left.121^{\circ} \mathrm{C}, 2 \mathrm{~h}\right)$.

The AM fungus, Funneliformis mosseae (T.H. Nicolson \& Gerd.) C. Walker \& A. Schüßler was propagated through the trap culture with white clover for 16 weeks. Mycorrhizal treatment was applied at 1500 spores per pot at transplanting. These spores were added in the plant rhizosphere. Same amount of autoclaved inocula was applied into the non-AMF pot. The experiment was conducted in a glasshouse with $982 \mu \mathrm{mol} / \mathrm{m}^{2} / \mathrm{s}$ photonflux density, $27 / 20^{\circ} \mathrm{C}$ day $/$ night temperature, and $80 \%$ relative air humidity. To decrease growth environments, these plants were weekly relocated. Distilled water was supplied daily to maintain gravimetrical $75 \%$ of the maximum water holding capacity of the substrate until water stress was started.

After 16 weeks of AMF inoculation, two soil water treatments were applied: gravimetrical $75 \%$ (WW) and $55 \%$ (DS) of the maximum water holding capacity of the substrate, respectively. The soil water treatments were maintained for 6 weeks until plant harvest.

The experiment consisted of soil water treatments (DS and $\mathrm{WW}$ ) and AMF treatments (+AMF and -AMF), which are WW+AMF, WW-AMF, DS+AMF, and DS-AMF, respectively. Each treatment was replicated five times, resulting in a total of 20 pots.

\section{Determinations of variables}

The fresh weights of AM and non-AM plants were measured after harvest, and divided into shoots and roots. The numbers of different-order lateral roots were counted. The intact root system of each plant was quickly scanned with an Epson Flatbed Scanner (J221A, Indonesia). The scanned root pictures were analyzed by the WinRHIZO software (Regent Instruments Inc., Quebec, Canada), and the root morphological traits, including total length, surface area, projected area, average diameter, and volume were obtained.

The 1-cm-long root samples were cleared in a $10 \%(\mathrm{w} / \mathrm{v})$ $\mathrm{KOH}$ solution and stained with $0.05 \%(\mathrm{w} / \mathrm{v})$ trypan blue according to Phillips and Hayman (1970). Mycorrhizal colonization was calculated with the following formula: $\mathrm{AM}$ colonization $(\%)=\mathrm{AM}$ root intercepts/total root intercepts $\times 100$.

Leaf water potential (leaf $\Psi$ ) was recorded before the plant harvest using a PSYPRO Water Potential System with a leaf hygrometer (L-51A-SF, WESCOR).

The protocol for extraction of root phytohormones was done by Chen et al. (2009). The frozen root samples with $0.2 \mathrm{~g}$ were ground with $4 \mathrm{~mL} 80 \%$ methanol containing $1 \mathrm{mmol} / \mathrm{L} 2,6$-ditert-butyl-4-methylphenol. The extraction was incubated at $4^{\circ} \mathrm{C}$ for $4 \mathrm{~h}$ and centrifuged at $4,000 \mathrm{~g}$ for $10 \mathrm{~min}$ at $4^{\circ} \mathrm{C}$. The supernatants were passed through AccuBond C18 solid phase extraction cartridge (Agilent Technologies Inc., USA) and dried under $\mathrm{N}_{2}$. The residues were dissolved in $2 \mathrm{~mL}$ phosphate buffer containing $0.1 \%$ Tween 20 and $0.1 \%$ gelatin $(\mathrm{pH} 7.5)$ for phytohormone analysis. Quantification of root IAA, GA, BRs, methyl jasmonate (MeJA), ABA, and zeatin riboside (ZR) by ELISA was described by Yang et al. (2001). These ELISA kits were produced by the Crop Chemical Control Center, China Agricultural University, Beijing, China.

\section{Statistical analysis}

Data were subjected to analysis of one-factor variance (ANOVA) in SAS (SAS Institute Inc., Cary, NC, USA) and followed by the Duncan's multiple range tests $(P<0.05)$ to compare the significant differences between these treatments.

\section{RESULTS AND DISCUSSION}

\section{Root AM colonization}

In this study, there was not root mycorrhizal colonization in the non-AMF treated plants. The root AM colonization 
in the inoculated seedlings varied from $53.5 \pm 4.8 \%$ under the WW to $38.7+5.2 \%$ under the DS. The DS treatment significantly decreased root colonization by $27.7 \%$, as compared with the WW treatment. Such reduction in root AM colonization by the DS may be due to relatively lower germination of AMF spores, lower carbohydrate supply from the host plant (Wu et al., 2013), and the decrease of root exudates (Fagbola et al., 2001).

\section{Total plant biomass}

The present work indicated that compared with the WW, the DS significantly restricted total plant biomass production, irrespective of $\mathrm{AM}$ and non-AM seedlings (Fig. 1). However, AMF inoculation notably increased total plant biomass by 159 and $111 \%$ under the WW and the DS conditions, respectively. This is in agreement with the findings of Asrar et al. (2013), who reported that regardless of DS level, the snapdragon plants colonized by Glomus deserticola had a significantly higher plant biomass than non-AMF colonized plants. The growth enhance by mycorrhization with F. mosseae was observed in trifoliate orange seedlings under different phosphorus substrate levels (Wu et al., 2015). The enhancement of growth in AM plants is often related to improvement of nutrient acquisition, especially P (Asrar et al., 2013), and direct water uptake and transport by extraradical hyphae (Abbaspour et al., 2012; Zou et al., 2015).

\section{Leaf water potential (leaf $\Psi$ )}

As stated by Davies et al. (1992), extraradical hyphae could improve drought tolerance by facilitating soil water uptake. Hyphal tips are hydrophilic, and thereby soil water can be taken up at the tips and transported from tips to

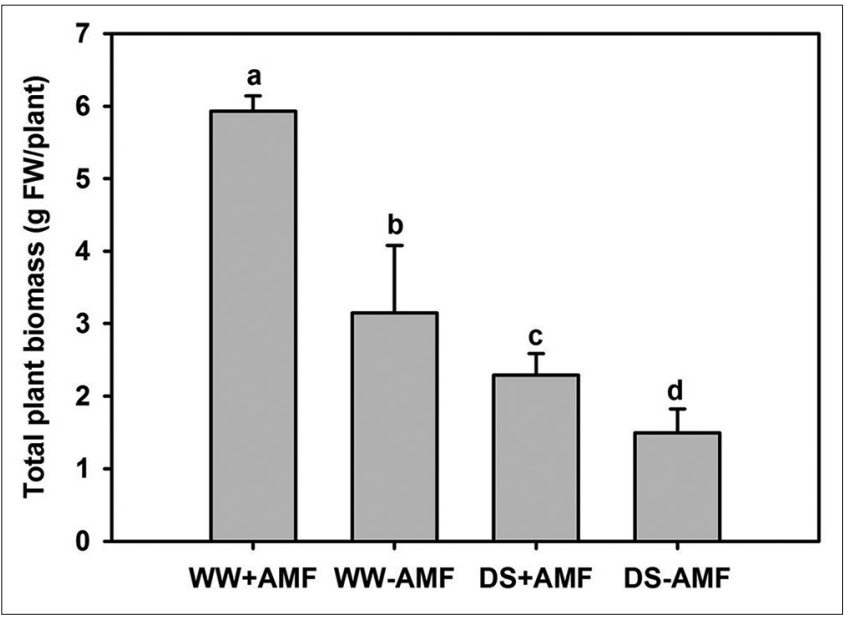

Fig 1. Effect of AMF and drought stress on total plant biomass of trifoliate orange seedlings. Data (means $\pm \mathrm{SD}, n=5$ ) followed by different letters above the bars among treatments indicate significant differences at the $5 \%$ level. Abbreviation: +AMF: inoculation with Funneliformis mosseae; -AMF: inoculation without Funneliformis mosseae; WW: well-watered; DS: drought stress root cortical cells, without encountering a membrane (Allen, 2007). On the other hand, mycorrhizal hyphae would extend soil water depletion zones, thus, providing mycorrhizal roots more access to available soil water (Wu et al., 2013; Zou et al., 2015). Furtherly, mycorrhizal hyphae enhance the ratio of below-ground absorptive surface to leaf area (Asrar et al., 2012). This may explain that in the present study, a significantly higher leaf $\Psi$ was found in AM trifoliate orange than in non-AM trifoliate orange exposed to WW and DS (Fig. 2).

\section{Root morphological modification}

Root systems have several functions, such as storage of carbohydrates and nutrients and transport of nutrients and water (Comas et al., 2013). In the trifoliate orange, compared with the WW treatment, the 6-week DS treatment considerably inhibited root total length, projected area, surface area, average diameter, and volume, which was more significant in AM plants than in non-AM plants (Table 1). Possibly, greater plant growth in AM plants was strongly affected by DS than those in non-AM plants. When the trifoliate orange plant was colonized by Funneliformis mosseae, the AM plants exhibited better root morphological traits than the non-AM plants, irrespective of WW or DS treatment (Table 1). Moreover, the AM plants also induced more number of first, second and third order lateral roots than the non-AM plants exposed to WW and DS. This is consistent with the results described by Orfanoudakis et al. (2010) in Alnus glutinosa plants. As a result, greater root morphological traits in AM plants would provide more exploration of soil volume for water and nutrient acquisition under the DS (Comas et al., 2013), which is the important mechanism to enhance drought tolerance in the host plant.

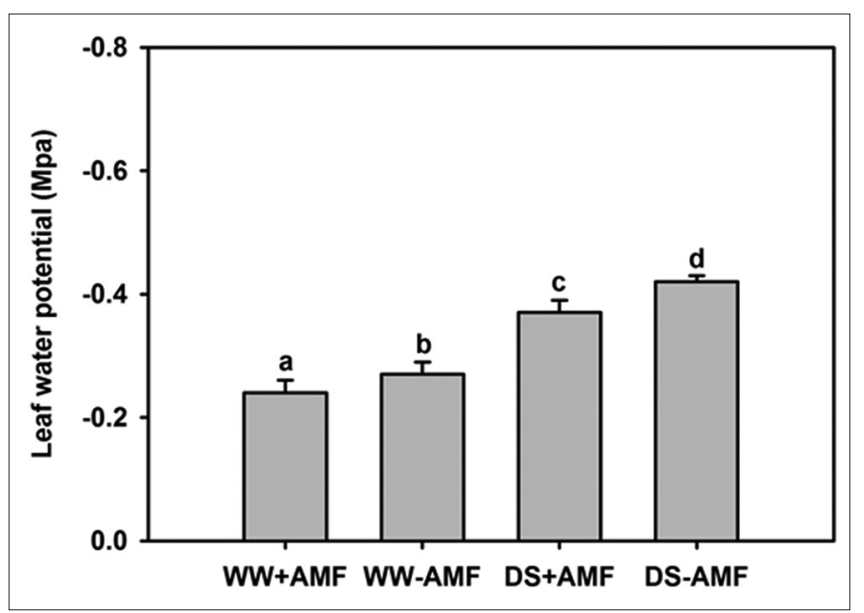

Fig 2. Effect of $A M F$ and drought stress on leaf water potential of trifoliate orange seedlings. Data (means $\pm \mathrm{SD}, n=5$ ) followed by different letters above the bars among treatments indicate significant differences at the $5 \%$ level. Abbreviation: +AMF: inoculation with Funneliformis mosseae; -AMF: inoculation without Funneliformis mosseae; WW: well-watered; DS: drought stress. 
Table 1: Effects of AMF and drought stress on root morphological traits and lateral root number of trifoliate orange seedlings

\begin{tabular}{|c|c|c|c|c|c|c|c|c|}
\hline \multirow[t]{2}{*}{ Treatments } & \multirow{2}{*}{$\begin{array}{l}\text { Total length } \\
\text { (cm) }\end{array}$} & \multirow{2}{*}{$\begin{array}{l}\text { Projected } \\
\text { area }\left(\mathrm{cm}^{2}\right)\end{array}$} & \multirow{2}{*}{$\begin{array}{c}\text { Surface } \\
\text { area }\left(\mathrm{cm}^{2}\right)\end{array}$} & \multirow{2}{*}{$\begin{array}{l}\text { Average diameter } \\
(\mathrm{mm})\end{array}$} & \multirow{2}{*}{$\begin{array}{l}\text { Volume } \\
\left(\mathrm{cm}^{3}\right)\end{array}$} & \multicolumn{3}{|c|}{ Number of lateral root } \\
\hline & & & & & & $1^{\text {st }}$ & $2^{\text {nd }}$ & $3^{\text {rd }}$ \\
\hline WW+AMF & $576 \pm 69 a$ & $28.8 \pm 4.9 a$ & $90.5 \pm 15.5 a$ & $0.50 \pm 0.02 a$ & $1.13 \pm 0.26 a$ & $49 \pm 3 a$ & $248 \pm 21 a$ & $15 \pm 2 a$ \\
\hline WW-AMF & $395 \pm 39 b c$ & $16.8 \pm 1.7 \mathrm{bc}$ & $52.8 \pm 5.4 \mathrm{bc}$ & $0.43 \pm 0.02 \mathrm{c}$ & $0.56 \pm 0.07 b c$ & $41 \pm 5 b$ & $140 \pm 13 b$ & $6 \pm 2 c$ \\
\hline DS+AMF & $447 \pm 116 b$ & $20.3 \pm 3.4 b$ & $63.8 \pm 10.7 \mathrm{~b}$ & $0.46 \pm 0.02 b$ & $0.73 \pm 0.06 b$ & $44 \pm 3 b$ & $159 \pm 17 b$ & $9 \pm 2 b$ \\
\hline DS-AMF & $280 \pm 108 c$ & $12.4 \pm 5.0 c$ & $39.0 \pm 15.8 \mathrm{c}$ & $0.43 \pm 0.01 \mathrm{c}$ & $0.44 \pm 0.19 c$ & $36 \pm 4 c$ & $106 \pm 7 c$ & $2 \pm 1 d$ \\
\hline
\end{tabular}

Data (means $\pm S D, n=5$ ) followed by different letters between treatments indicate significant differences at $5 \%$ level. Abbreviation: + AMF: Inoculation with Funneliformis mosseae; -AMF: Inoculation without Funneliformis mosseae; WW: Well-watered; DS: Drought stress

\section{Changes in root endogenous hormones}

In this study, six endogenous hormones, including IAA, GAs, BR, ABA, MeJA, and ZR were identified (Fig. 3).

\section{ABA}

Evidence showed that $\mathrm{ABA}$ has the key role in stress signal network and can mediate stress-induced stomatal closure (Tuteja, 2007). The present study indicated that the DS treatment strongly stimulated the increase of root ABA concentration in AM and non-AM seedlings (Fig. 3). Compared with non-AMF treatment, AMF inoculation also increased root ABA level by 13.6 and $8.4 \%$ under the WW and the DS conditions, respectively. Greater root ABA levels in AM roots suggested that AM plants possessed higher drought tolerance in regulating plant water status via guard cells and induction of genes encoding enzymes and proteins (Zhang et al., 2006).

\section{IAA}

Root IAA level was significantly decreased by the DS treatment, irrespective of AMF inoculated or not (Fig. 3). It is in agreement with the results of Man et al. (2011) in two tall fescue cultivars exposed to DS. The AMF inoculation represented a significantly higher root IAA level than the non-AMF control, regardless of soil water stressed or not. In general, IAA is positively associated with drought tolerance (Zhang et al., 2009). Moreover, IAA is closely related to root growth (Nordstrom et al., 1991). Therefore, this result suggests that IAA changes induced by mycorrhization are an important mechanism involved in greater root morphology and drought tolerance.

\section{ZR}

CTK is an antagonist to ABA, and drought treatment often results in decreased levels of CTK (Peleg and Blumwald, 2011). This study indicated that the DS treatment significantly decreased root ZR level than the WW treatment (Fig. 3). Meanwhile, the AMF treatment notably increased root ZR concentration by 79.4 and $55.9 \%$ under the WW and the DS conditions, respectively. This is in agreement with the results of Drüge and Schönbeck (1992) in flax plants. Higher ZR concentration in stressed AM roots suggests that AMs enhanced sink strength and promoted photosynthesis in the host plant (Novakova et al., 2007).

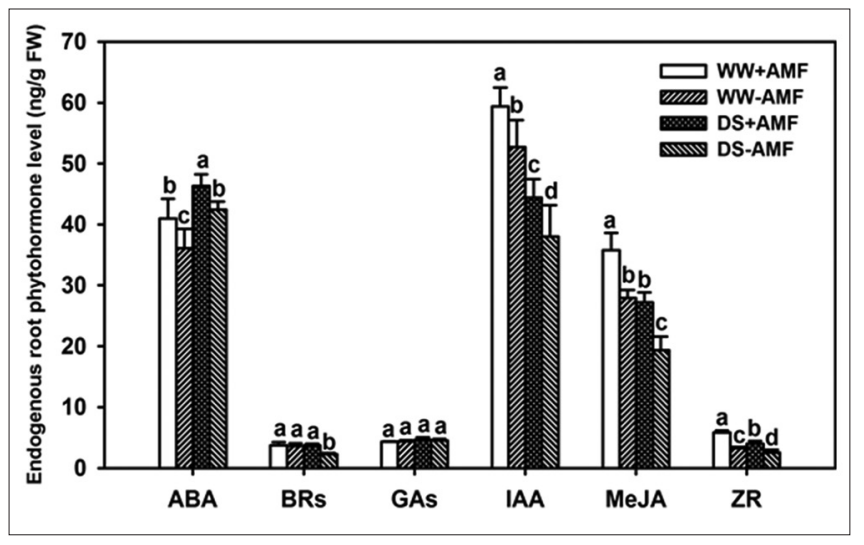

Fig 3. Effect of AMF and drought stress on root endogenous phytohormone concentrations of trifoliate orange seedlings. Data (means $\pm \mathrm{SD}, n=4$ ) followed by different letters above the bars among treatments indicate significant differences at the $5 \%$ level. Abbreviation: +AMF: inoculation with Funneliformis mosseae; -AMF: inoculation without Funneliformis mosseae; WW: well-watered; DS: drought stress.

\section{MeJA}

MeJA has been identified as a vital cellular regulator that mediates diverse plant developmental processes and defense responses against biotic and abiotic stresses (Cheong and Choi, 2003). The present study indicated that the 6-week DS treatment resulted in a significant decrease of root MeJA concentration in AM and non-AM seedlings, as compared with the WW control (Fig. 3). The AM seedlings had 28.2 and 40.4\% significantly higher root MeJA level than the non-AM seedlings under the WW and the DS conditions, respectively. The increase of MeJA by mycorrhization, in this study, is in agreement with the previous results as shown for several plant species (Hause et al., 2002; Meixner et al., 2005). As stated by LudwigMüller (2010), JA contributed to the life cycle of the AM fungus. Moreover, the up-regulation of JA biosynthesis took place in arbuscule-containing root cells of Medicago truncatula (Isayenkov et al., 2005). As a result, AMF may strongly induce the upregulation of JA biosynthetic genes involved in the DS response.

\section{BRs}

BRs are a group of naturally occurring plant steroidal compounds, who have the characteristics of the enhancement of stress tolerance through the cross-talk between BRs and other hormones or BR-induced complex 
molecular changes (Krishna, 2003). Our study indicated that application of the DS did not affect the change of root BR in AM plants, but significantly decreased root BR level in non-AM plants (Fig. 3). On the other hand, AM roots recorded significantly higher BR levels only under the DS, but not under the WW conditions, indicating greater drought tolerance in AM plants.

\section{GAs}

GAs are an essential for many plant developmental processes, including seed germination, stem elongation, leaf expansion, trichome development, pollen maturation and the induction of flowering (Achard and Genschik, 2009). In this work, the DS stress did not significantly alter the root GAs level, as compared with the WW treatment, irrespective of AM or non-AM plants (Fig. 3). Similarly, the mycorrhizated treatment had not significant changes in root GAs level under the WW and the DS conditions. It implies that AMs did not provide any influences on the root GAs level.

\section{CONCLUSIONS}

In short, the 6-week drought treatment resulted in the decrease in root morphology, while the AMF inoculation significantly enhanced the root morphological traits, irrespective of drought stress or not. On the other hand, the AMF inoculation positively stimulated the levels of 'positive growth regulators', including IAA and ZR, and the concentrations of 'stress hormones', including ABA, $\mathrm{BRs}$, and MeJA, who strongly enhanced drought tolerance in AM plants.

\section{ACKNOWLEDGEMENT}

The present study was supported by the National Natural Science Foundation of China (31101513) and the Creative Experimental Project of National Undergraduate Students (2014043).

\section{Author's contributions}

C. G and Y.N. Z designed the study and took the data. J. L, Z.L. C, J.D. H, and Y.N. Z did the data analysis and wrote this paper. Y.N. Z supervised the research project and also provided critical revision.

\section{REFERENCES}

Abbaspour, H., S. Saeidi-Sar, H. Afshari and M. A. Abdel-Wahhab. 2012. Tolerance of mycorrhiza infected pistachio (Pistacia vera L.) seedling to drought stress under glasshouse conditions. J. Plant Physiol. 169: 704-709.

Achard, P. and P. Genschik. 2009. Releasing the brakes of plant growth: How Gas shutdown DELLA proteins. J. Exp. Bot. 60: 1085-1092.
Allen, M.F. 2007. Mycorrhizal fungi: Highways for water and nutrients in arid soils. Vadose ZJ. 6: 291-297.

Asrar, A. A., G. M. Abdel-Fattah and K. M. Elhindi. 2012. Improving growth, flower yield, and water relations of snapdragon (Antirhinum majus L.) plants grown under well-watered and water-stress conditions using arbuscular mycorrhizal fungi. Photosynthetica. 50: 305-316.

Chen, Q., W. B. Qi, R. J. Reiter, W. Wei and B. M. Wang. 2009. Exogenously applied melatonin stimulates root growth and raises endogenous indoleacetic acid in roots of etiolated seedlings of Brassica juncea. J. Plant Physiol. 166: 324-328.

Cheong, J. J. and Y. D. Choi. 2003. Methyl jasmonate as a vital substance in plants. Trends Genet. 19: 409-413.

Comas, L. H., S. R. Becker, V. M. V. Cruz, P. F. Byrne and D. A. Dierig. 2013. Root traits contributing to plant productivity under drought. Front. Plant Sci. 4: 442.

Cruz, A. F., T. Ishii and K. Kadoya. 2000. Effects of arbuscular mycorrhizal fungi on tree growth, leaf water potential, and levels of 1-aminocyclopropane-1-carboxylic acid and ethylene in the roots of papaya under water-stress conditions. Mycorrhiza. 10: 121-123.

Davies, F. T., J. Potter and R. G. Linderman. 1992. Mycorrhiza and repeated drought exposure affect drought resistance and extraradical hyphae development of pepper plants independent of plant size and nutrient content. J. Plant Physiol. 139: 289-294.

Diego, N. D., I. Saiz-Fernandez, J. L. Rodriguez, P. Perez-Alfocea, M. C. Sampedro, R. J. Barrio, M. Bacuesta and P. Moncalean. 2015. Metabolites and hormones are involved in the intraspecific variability of drought hardening in radiata pine. J. Plant Physiol. 188: 64-71.

Drüge, U. and F. Schönbeck. 1992. Effect of vesicular-arbuscular mycorrhizal. infection on transpiration, photosynthesis and growth of flax (Linum usitissimum L.) in relation to cytokinin levels. J. Plant Physiol. 141: 40-48.

Fagbola, O., O. Osonubi, K. Mulongoy and S. A. Odunfa. 2001. Effects of drought stress and arbuscular mycorrhiza on the growth of Gliricidia sepium (Jacq). Walp, and Leucaena leucocephala (Lam.) de Wit. in simulated eroded soil conditions. Mycorrhiza. 11: 215-223.

Fahad, S., S. Hussain, A. Matloob, F. A. Khan, A. Khaliq, S. Saud, S. Hassan, D. Shan, F. Khan, N. Ullah, M. Faiq, M. R. Khan, A. K. Tareen, A. Khan, A. Ullah, N. Ullah and J. L. Huang. 2014. Phytohormones and plant responses to salinity stress: A review. Plant Growth Regul. 75: 391-404.

Goicoechea, N., M. C. Antolin and M. Sanchez-Diaz. 1997. Gas exchange is related to the hormone balance in mycorrhizal or nitrogen-fixing alfalfa subjected to drought. Physiol. Plant. 100: 989-997.

Hause, B., W. Maier, O. Miersch, R. Kramell and D. Strack. 2002. Induction of jasmonate biosynthesis in arbuscular mycorrhizal barley roots. Plant Physiol. 130: 1213-1220.

Isayenkov, S., C. Mrosk, I. Stenzel, D. Strack and B. Hause. 2005. Suppression of allene oxide cyclase in hairy roots of Medicago truncatula reduces jasmonate levels and the degree of mycorrhization with Glomus intraradices. Plant Physiol. 139: 1401-1410.

Johnson, D. and L. Gibert. 2015. Interplant signalling through hyphal networks. New Phytol. 205: 1148-1153.

Kovosá, K., I. T. Prasil, P. Vitamvas, P. Dobrev, V. Motyka, K. Flokova, O. Novak, V. Tureckova, J. Rolcik, B. Pesek, A. Travnickova, A. Gaudinova, G. Galiba, T. Janda, E. Vlasakova, P. Prasilova and R. Vankova. 2012. Complex phytohormone responses during the cold acclimation of two wheat cultivars differing in cold 
tolerance, winter Samanta and spring Sandra. J. Plant Physiol. 169: 567-576.

Krishna, P. 2003. Brassinosteroid-mediated stress responses. J. Plant Growth Regul. 22: 289-297.

Ludwig-Müller, J. 2010. Hormonal responses in host plants triggered by arbuscular mycorrhizal fungi. In: Koltai, H. and Y. Kapulnik (Eds.), Arbuscular Mycorrhizas: Physiology and Function. Springer Science Business, Media B.V., New York, Pp. 169-196.

Man, D., Y. X. Bao, L. B. Han and X. Z. Zhang. 2011. Drought tolerance associated with proline and hormone metabolism in two tall fescue cultivars. HortScience. 46: 1027-1032.

Meixner, C., J. Ludwig-Müller, O. Miersch, P. Gresshoff, C. Staehelin and $\mathrm{H}$. Vierheilig. 2005. Lack of mycorrhizal autoregulation and phytohormonal changes in the supernodulating soybean mutant nts1007. Planta. 222: 709-715.

Nazar, R., S. Umar, N. A. Khan and O. Sareer. 2015. Salicylic acid supplementation improves photosynthesis and growth in mustard through changes in proline accumulation and ethylene formation under drought stress. South Afr. J. Bot. 98: 84-94.

Nordstrom, A., F. A. Jacobs and L. Eliasson. 1991. Effect of exogenous indole-3-acetic acid and indole-3-butyric acid on internal levels of the respective auxins and their conjugation with aspartic acid during adventitious root formation in pea cuttings. Plant Physiol. 96: 856-861.

Novakova, M., P. Dobrev, V. Motyka, A. Gaudinova, J. Malbeck, J. Pospisilova, D. Haisel, H. Storchova, J. Dobra, M. C. Mok, D. W. S. Mok, R. Martin and R. Vankova. 2007. Cytokinin function in drought stress response and subsequent recovery. In: Xu, Z. H., J. Y. Li, Y. B. Xue and W. C. Yang (Eds.), Biotechnology and Sustainable Agriculture 2006 and Beyond. Springer, Netherlands, Pp. 171-174.

Orfanoudakis, M., C. T. Wheeler and J. E. Hooker. 2010. Both the arbuscular mycorrhizal fungus Gigaspora rosea and Frankia increase root system branching and reduce root hair frequency in Alnus glutinosa. Mycorrhiza. 20: 117-126.

Peleg, Z. and E. Blumwald. 2011. Hormone balance and abiotic stress tolerance in crop plants. Curr. Opin. Plant Biol. 14: 290-295.

Phillips, J. M. and D. S. Hayman.1970. Improved procedures for clearing roots and staining parasitic and vesicular-arbuscular mycorrhizal fungi for rapid assessment of infection. Trans. Br. Mycol. Soc. 55: 158-161.

Shan, L. S., C. H. Yang, Y. Li, Y. N. Duan, D. M. Geng, Z. Y. Li, R. Zhang, G. F. Duan and Ж. А. Васильевич. 2015. Effects of drought stress on root physiological traits and root biomass allocation of Reaumuria soongorica. Acta Ecol. Sin. 35: 155-159.

Subramanian, K. S. and C. Charest. 1998. Arbuscular mycorrhizae and nitrogen assimilation in maize after drought and recovery. Physiol. Plant. 102: 285-296.

Tuteja, N. 2007. Abscisic acid and abiotic stress signaling. Plant Signal Behav. 2: 135-138.

Wang, S., A. K. Srivastava, Q. S. Wu and R. Fokom. 2014. The effect of mycorrhizal inoculation on the rhizosphere properties of trifoliate orange (Poncirus trifoliata L. Raf.). Sci. Hortic. 170: 137-142.

Wu, Q. S., A. K. Srivastava and Y. N. Zou. 2013. AMF-induced tolerance to drought stress in citrus: A review. Sci. Hortic. 164: 77-87.

Wu, Q. S., A. K. Srivastava and Y. Li. 2015. Effect of mycorrhizal symbiosis on growth behavior and carbohdyrate metabolism of trifoliate orange under different substrate $\mathrm{P}$ levels. J. Plant Growth Regul. 34: 495-508.

Yang, J. C., J. H. Zhan, Z. Q. Wang, Q. S. Zhu and W. Wang. 2001. Hormonal changes in the grains of rice subjected to water stress during grain filling. Plant Physiol. 127: 315-323.

Zhang, J. H., W. S. Jia, J. C. Yang and A. M. Ismail. 2006. Role of ABA in integrating plant responses to drought and salt stresses. Field Crops Res. 97: 111-119.

Zhang, X., E. H. Ervin, G. K. Evanylo and K. C. Haering. 2009. Impact of biosolids on hormone metabolism in drought-stressed tall fescue. Crop Sci. 49: 1893-1901.

Zhang, Z. Z., Y. G. Lou, D. J. Deng, M. M. Rahman and Q. S. Wu. 2015. Effects of common mycorrhizal network on plant carbohydrates and soil properties in trifoliate orange-white clover association. PLoS One. 10: e0142371.

Zou, Y. N., A. K. Srivastava, Q. D. Ni and Q. S. Wu. 2015. Disruption of mycorrhizal extraradical mycelium and changes in leaf water status and soil aggregate stability in rootbox-grown trifoliate orange. Front. Microbiol. 6: 203. 